
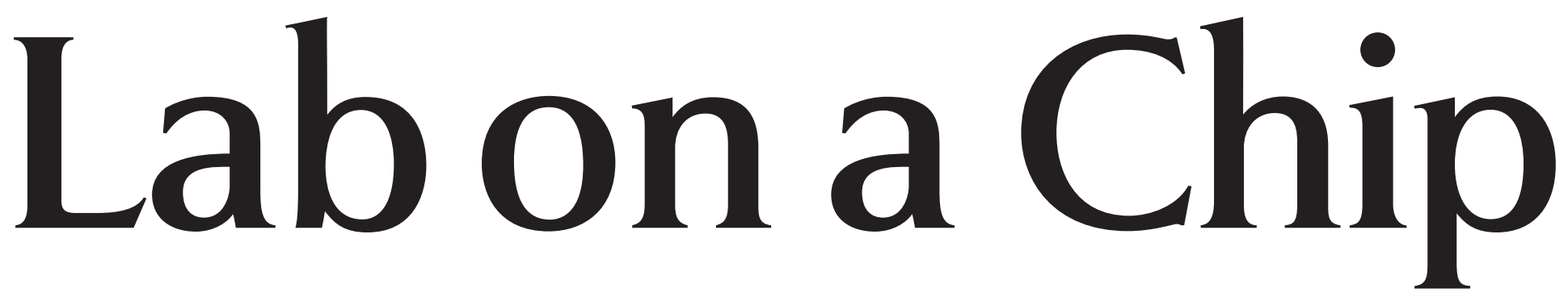

Miniaturisation for chemistry, physics, biology, materials science and bioengineering
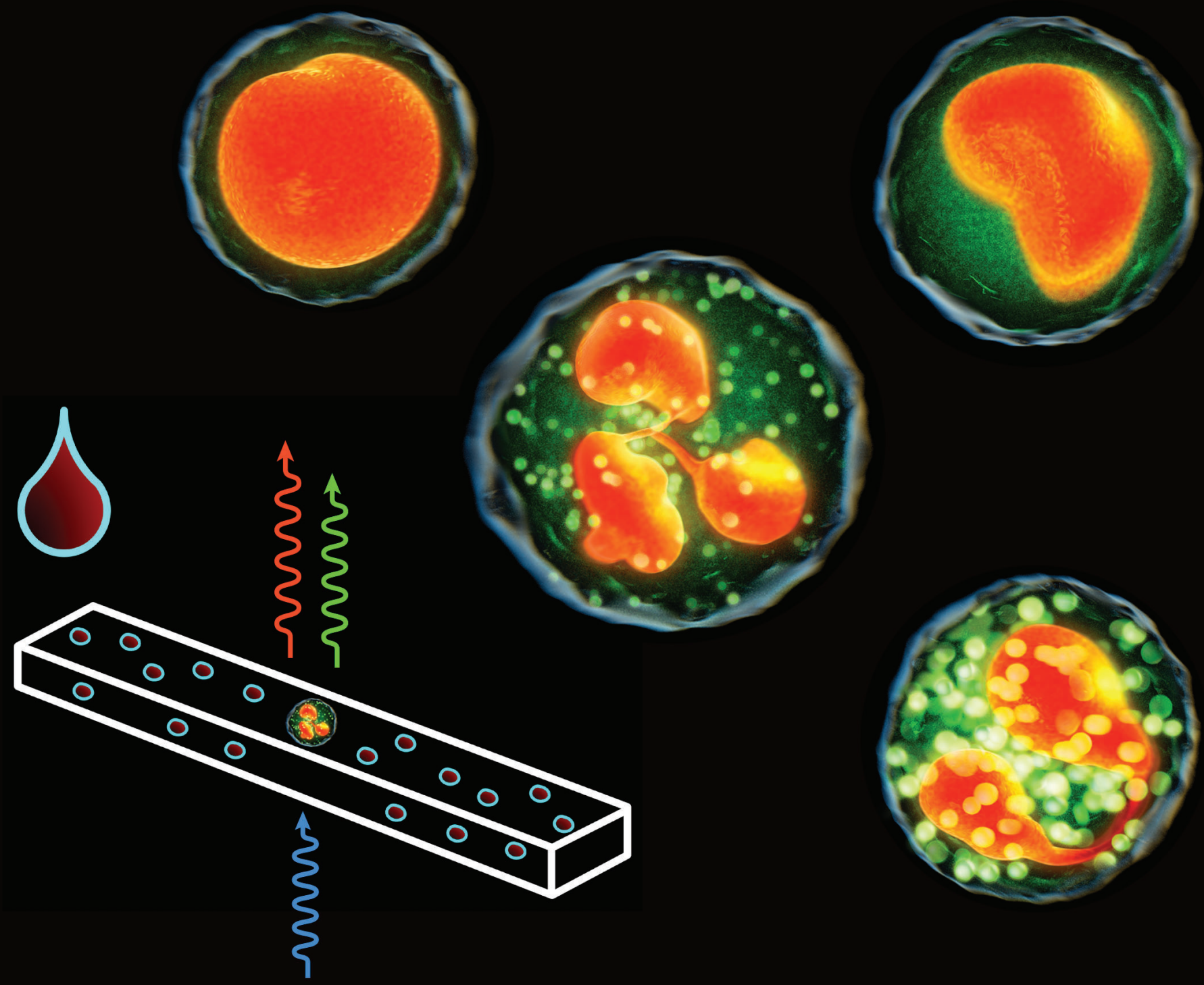


\title{
PAPER
}

Cite this: Lab Chip, 2013, 13, 1257

\section{Four-part leukocyte differential count based on sheathless microflow cytometer and fluorescent dye assay $\dagger$}

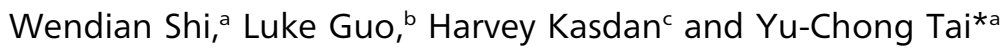

Received 19th September 2012, Accepted 17th January 2013

DOI: $10.1039 / c 3 \mid c 41059 e$

www.rsc.org/loc

\begin{abstract}
Leukocyte differential count is one of the most frequently ordered clinical tests in hospitals. This paper reports a point-of-care test for the leukocyte count by using a microflow cytometer and a fluorescent dye assay. The dye assay relied on fluorescent detection alone to count leukocytes in blood and to identify leukocyte subtypes. By combining the fluorescent assay with a sheathless microflow design, the proposed method achieved a minimal sample volume by eliminating excessive dilution and sheath flow. In this paper, a four-part leukocyte differential count including lymphocyte, monocyte, neutrophil and eosinophil was demonstrated, and the whole test consumed only a small amount of blood $(5 \mu \mathrm{L})$ and reagents $(68 \mu \mathrm{L}$ in total). The merits of minimal sample volume, long reagent shelf life and portable instrument made this method optimal for point-of-care applications.
\end{abstract}

\section{Introduction}

Leukocyte (i.e. white blood cell) count is one of the most frequently ordered clinical tests in hospital. ${ }^{1}$ This test enumerates leukocyte cells $\left(4-11 \times 10^{3}\right.$ cells per $\left.\mu \mathrm{L}\right)$ in blood, reporting the total count of leukocytes and the differential count of leukocyte subtypes. Clinical laboratories normally report the leukocyte count with either a three-part differential (lymphocyte, monocyte and neutrophil), or a complete fivepart differential (lymphocyte, monocyte, neutrophil, eosinophil and basophil). The test results, particularly the differential count results, are important in clinical diagnosis of diseases such as infections (virus, bacteria, parasite, fungi, etc.), inflammation, allergies, etc.

In recent years, there are many emerging needs for a pointof-care test of the leukocyte count. ${ }^{2}$ The NASA space program ${ }^{3}$ is looking for technology that enables inflight leukocyte count to monitor astronauts' health status in the space environment. For example, astronauts' immune systems are known to weaken during long-term spaceflight, and this change could be closely monitored by the leukocyte count test. In addition, a

${ }^{a}$ Caltech Micromachining Laboratory, Department of Electrical Engineering, California Institute of Technology, Pasadena, California, 91125, USA. E-mail: yctai@its.caltech.edu; Fax: +1 626-395-8475; Tel: +1 626-395-8317 ${ }^{b}$ Department of Electrical Engineering and Computer Science, Massachusetts Institute of Technology, Cambridge, Massachusetts, 02139, USA

${ }^{c}$ LeukoDx Inc., 3 Hamarpe St., POB 45409, Jerusalem, 91451, Israel

$\dagger$ Electronic supplementary information (ESI) available: The estimation of coincidence, the description of the microfluidic device and the cytometer system, microscopy quantification of leukocytes' fluorescence, calibration by fluorescent beads and the comparison of two dye assays. See DOI: 10.1039/ c3lc41059e point-of-care leukocyte count is also beneficial for applications on earth to provide on-site diagnosis. For example, such a test is useful in physicians' offices to provide rapid screening to distinguish between viral and bacterial infections. Applications of the point-of-care leukocyte count in the fields of telemedicine, out-patient monitoring and medical care in remote areas have also been previously reviewed. ${ }^{2}$

However, presently the leukocyte count test is mainly performed in central clinical laboratories. The state-of-the-art technologies, such as hematology analyzer and flow cytometer, normally require bulky instruments and consume large amount of reagents, therefore are not suitable for point-ofcare applications. To solve this problem, there have been increasing efforts to miniaturize the leukocyte count test. ${ }^{4-6}$ Altendorf et $a l^{4}$ first attempted counting blood cells in a microfluidic channel and detecting leukocytes by light scattering measurement. Seo et $a l .{ }^{5}$ developed a lensless microscope for blood cell imaging and reported the count of leukocytes among other blood cells. Holmes et al. ${ }^{6}$ counted leukocyte cells in a microfluidic channel by measuring electrical impedances, and demonstrated a leukocyte count with the three-part differential.

Meanwhile, this project is focusing on microflow cytometry, which is readily considered as a promising approach to miniaturize cell analysis. ${ }^{7-13}$ Cell analysis on flow cytometers typically involves two steps: ${ }^{14}$ first, labeling target cells with detection assays, e.g., fluorophore-conjugated antibodies, and second, detecting target cells by corresponding optical signals, e.g., fluorescence. The same principle has been applied to "microflow" cytometers for leukocyte analysis. Chan et al. ${ }^{8}$ investigated the protein expressions on leukocyte cells in a 
microflow cytometer; the works of Wang et al., ${ }^{9}$ Yun et al. ${ }^{10}$ and Herr et al. ${ }^{11}$ studied the count of two lymphocyte subtypes, CD4+ and CD8+; and the works of Kummrow et al. ${ }^{12}$ and Frankowski et al. ${ }^{13}$ reported the three-part differential count of leukocyte on a microfluidic-based cytometer.

Many efforts have been focused on optimizing the fluidic designs of the microflow cytometers. ${ }^{15-20}$ Nevertheless, for point-of-care applications, it is equally important to optimize the detection assays. The previous reports ${ }^{8-13}$ have mostly used assays of fluorophore-conjugated antibodies for leukocyte analysis on the microflow cytometers. However, the low temperature needed for reagent storage makes these assays less than ideal for point-of-care applications, particularly in resource-limited settings. In comparison, assays of fluorescent dyes have been proven as useful alternatives in cell analysis. ${ }^{21}$ They are considered suitable for point-of-care applications because many fluorescent dyes can be stored at room temperature with long shelf life. In addition, assays of fluorescent dyes typically have the advantages of lower cost and higher signal intensities comparing to the assays of fluorophore conjugated antibodies.

Therefore, it is attractive to develop fluorescent dye assays for the leukocyte count test in microflow cytometers. In fact, we had previously reported the implementation of dye assays for the leukocyte count in microfluidic devices. ${ }^{22-25}$ For example, we implemented an assay of the fluorescent dye, Acridine Orange, in a microscope-based microflow cytometer, ${ }^{22}$ and a leukocyte count with a two-part differential (lymphocytes and non-lymphocytes) was achieved in whole blood samples. ${ }^{23}$ Meanwhile, in conventional flow cytometers, fluorescent dye assays have also been investigated for the leukocyte count test. ${ }^{26}$ For example, Adams et al. ${ }^{27}$ reported using the assay of Acridine Orange to demonstrate a leukocyte count with the three-part differential. Shapiro et $a{ }^{28}{ }^{28}$ developed an assay of a three dye mixture, i.e. Ethidium Bromide, Brilliant Sulfaflavine and a Stilbene Disulfonic Acid derivative, and demonstrated a leukocyte count with the five-part differential on a conventional flow cytometer. However, this assay required complex detection settings that were not suitable for portable applications. As a result, the development of a fluorescent dye assay suitable for microflow cytometers is still needed.

Here we report the implementation of a fluorescent dye assay on a microflow cytometer that achieves a leukocyte count with a four-part differential, including lymphocytes, monocytes, neutrophils and eosinophils. This assay detects leukocytes in blood by fluorescent signals, and the measurement is performed in a sheathless, microfluidic channel minimizing reagent volume. The whole system is enclosed in a suitcasesized package, which is suitable for point-of-care applications. The test results of the four-part differential count from this portable system closely match the results from tests performed in central clinical laboratories. In addition, the count of the fifth leukocyte subtype, basophil, is also investigated with this method.

\section{Materials and methods}

\section{Leukocyte detection with fluorescent dye assay}

The assay of this study consists of three fluorescent dyes including Propidium Iodide (PI), Fluorescein Isothiocyanate (FITC) and Basic Orange 21 (BO21). In this assay, leukocytes are selectively stained with these dyes, which introduce a high intensity of fluorescence to distinguish leukocytes from other blood cells.

Before measurement, blood samples are treated with the dye assay to introduce fluorescent staining. There are three major types of cells in human blood: leukocytes, erythrocytes and thrombocytes. $^{29}$ One feature distinguishing leukocytes from the other types of cells is their cellular nucleus, which can be selectively stained by the dye PI. After the assay treatment, leukocyte cells show a high intensity of fluorescence from the PI staining (Fig. 1-a). This is because PI molecules have high affinity binding to the nucleic acid contents (e.g. DNA) in the leukocyte nucleus. ${ }^{30}$ Meanwhile, the other two types of cells, erythrocytes and thrombocytes, have minimal fluorescence from the PI staining (Fig. 1-b). This is because the mature erythrocytes and thrombocytes lack nuclei. ${ }^{29}$ Therefore, leukocytes can be counted in blood samples by their high intensities of PI fluorescence measured in the microflow cytometer (Fig. 1-c).

This assay also contains two other fluorescent dyes, FITC and BO21. FITC is an amine-reactive dye, binding to proteins with high affinity. ${ }^{31}$ It is typically used in cell analysis for measuring the amount of cellular proteins. In this study, FITC staining is investigated as one of the fluorescence characteristics for identifying leukocyte subtypes. BO21 is normally used as a nucleic acid dye for the leukocyte staining. ${ }^{21} \mathrm{BO} 21$ is added in this assay because it was observed in our experiments that, as described later, $\mathrm{BO} 21$ helps to improve the differential between two leukocyte subtypes, lymphocyte and monocyte.

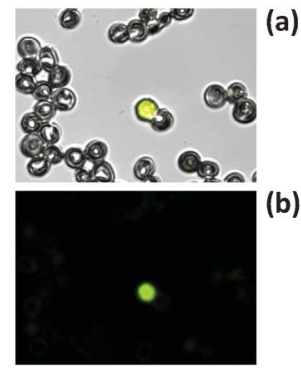

(a) Red Blood Cell White Blood Cell

(c)

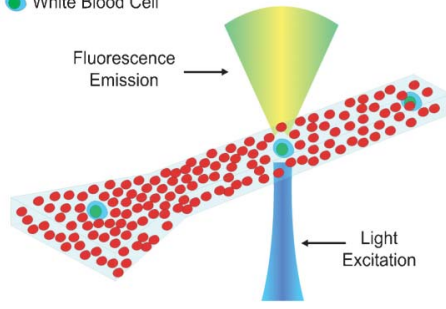

Fig. 1 (a) Bright-field, white-light microscope image of a leukocyte surrounded by erythrocytes and thrombocytes. The leukocyte is marked yellow for recognition in this picture. (b) Dark-field, fluorescence microscope image of the same cells. The leukocyte is clearly visible by the strong fluorescence, whereas the other cells remain dark. (c) Basic principle of the fluorescent detection of leukocyte cells in a sheathless microfluidic channel. Blood sample continuously flows through the channel, where the detection region is illuminated by the excitation light (marked blue), and the fluorescence emission is measured at the other side of the channel (marked yellow-green). 


\section{Leukocyte count in a sheathless channel}

As illustrated in Fig. 1-c, the microflow cytometer employs a sheathless channel for the fluorescence detection of the leukocytes. Leukocyte cells are counted one by one as the blood sample continuously flows through the channel. The sample is confined into a narrow stream by the microflow channel. Therefore, the coincidence of two or more leukocytes flowing through the detection channel together can be minimized.

To estimate this coincidence, blood samples are considered as fluid suspensions of single cells, which have an average number of $c$ leukocytes per volume blood. When this sample flows through a detection region of volume, $\Delta V$, the possibility of $n$ leukocytes flowing through together can be estimated by a Poisson process: ${ }^{32}$

$$
P(n)=(c \Delta V)^{n} \mathrm{e}^{-(c \Delta V) / n !, \quad n=0,1,2, \ldots}
$$

Therefore, the coincidence error, $P$, which is the possibility of two or more leukocytes flowing through together and being counted as one, ${ }^{32}$ can be expressed as:

$$
P=1-P(0)-P(1)=1-(1+c \Delta V) \mathrm{e}^{-c \Delta V}
$$

This coincidence error of the measurement limits the accuracy of the leukocyte count. ${ }^{32}$ The coincidence error can be reduced either by diluting the blood sample (equivalent to decreasing leukocyte concentration $c$ ), or by decreasing the detection region volume $\Delta V$, as shown in Fig. S1 ESI. $\dagger$

In this study, a rectangular shaped channel is used and the detection region has dimensions of $28 \mu \mathrm{m}$ in height, $32 \mu \mathrm{m}$ in width and $50 \mu \mathrm{m}$ in length. For blood samples with a typical concentration of leukocytes, e.g. $8 \times 10^{3}$ cells per $\mu \mathrm{L}$, the estimated coincidence error of the measurement is approximately 5\% without any dilution, and less than $0.01 \%$ with a 14 times dilution. The cross section of the sheathless channel in this design, $28 \mu \mathrm{m}$ in height and $32 \mu \mathrm{m}$ in width, is large enough so that blood cells (leukocytes 7-20 $\mu \mathrm{m}$, erythrocytes 6-8 $\mu \mathrm{m}$ and thrombocytes $2-3 \mu \mathrm{m}$ in diameter ${ }^{29}$ ) can flow through without jamming the detection channel.

\section{Microfluidic device and cytometer system}

A transparent PDMS microfluidic device, as shown in Fig. S2 ESI, $\dagger$ is used to provide the sheathless channel for the leukocyte count test. ${ }^{22}$ Fig. 2-a shows the sheathless channel on the device, which has a cross section of $28 \mu \mathrm{m}$ in height and $32 \mu \mathrm{m}$ in width, and a total length of $3 \mathrm{~mm}$. The $3 \mathrm{~mm}$ channel length is used to simplify the alignment between the fluidic channel and the optical illumination. The length of the detection region is determined by the size of the illumination area, which is approximately $50 \mu \mathrm{m}$ according to the size of the laser spot.

The cytometer system adopts an optical configuration of measuring two-color (green and red), laser-induced-fluorescence, as shown in Fig. 2-b. A $488 \mathrm{~nm}$ laser is used as the excitation source and focused on the sheathless channel of the microfluidic device by a condenser lens. The fluorescent emission is collected by another lens and passed through a $514 \mathrm{~nm}$ long pass filter to remove the residual excitation light.
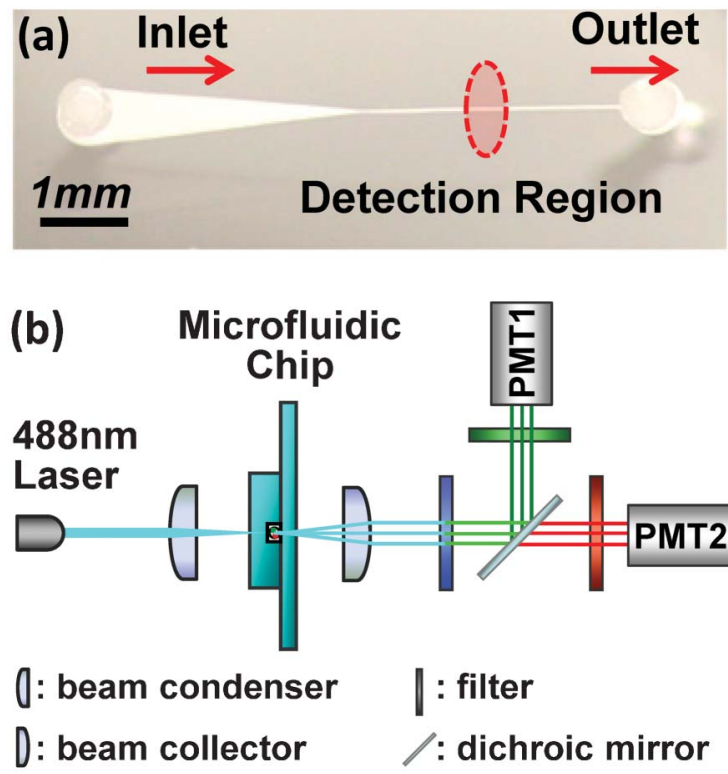

Fig. 2 (a) Zoom-in view of the sheathless channel for the optical detection. The red ellipse illustrates the detection region of the channel. (b) Illustration of the optical configuration for the laser-induced, two-color fluorescence detection.

A $593 \mathrm{~nm}$ dichroic mirror separates the collected fluorescence emission into two beams. The one with lower wavelength $(<593 \mathrm{~nm})$ is passed through a band pass filter $(520-550 \mathrm{~nm})$ and measured as the green fluorescence. The one with higher wavelength $(>593 \mathrm{~nm})$ is passed through a high pass filter (>600 $\mathrm{nm}$ ) and measured as the red fluorescence. In this configuration, the red fluorescence measures mainly the emission from the PI staining (emission peak $617 \mathrm{~nm}$ ) of the dye assay. Meanwhile, the green fluorescence measures the emissions from both the FITC staining (emission peak 520 $\mathrm{nm}$ ) and the BO21 staining (emission peak $525 \mathrm{~nm}$ ). The complete cytometer system is enclosed in a portable aluminum case $\left(12^{\prime \prime} \times 9^{\prime \prime} \times 5^{\prime \prime}\right)$, as shown in Fig. S3 ESI. $\dagger$

\section{Samples of whole blood and purified leukocyte subtypes}

Samples of human whole blood were purchased from HemaCare Corp., USA. These samples were venous blood obtained from healthy donors with informed consents, and kept in $5 \mathrm{ml}$ Vacutainer $^{\mathrm{TM}}$ tubes $\left(\mathrm{K}_{2}\right.$ EDTA, $7.2 \mathrm{mg}$, Becton Dickinson) in $4{ }^{\circ} \mathrm{C}$ refrigerator for storage. All experiments were carried out within $24 \mathrm{~h}$ of the blood drawing. For each sample, the test of a complete blood cell count was independently carried out in a clinical laboratory (USC Clinical Reference Laboratory, USA), and the results were used as reference data for the experiments.

Samples of leukocyte subtypes were prepared using the human whole blood and an immunomagnetic isolation kit, EasySep (Stemcell Technologies, Canada). The purification procedure has been previously reported ${ }^{33}$ and is briefly described here. First, the leukocyte fraction was prepared from the whole blood with a standard step of density gradient separation. In this step, $5 \mathrm{~mL}$ whole blood sample was mixed with $1 \mathrm{~mL}$ Hetasep buffer and centrifuged at $90 \mathrm{~g}$ at room 
temperature for $5 \mathrm{~min}$. Following the centrifugation, the supernatant layer containing the leukocyte fraction was collected, and washed twice using the Robosep buffer. Second, the leukocyte subtypes were purified from the leukocyte fraction with a step of immunomagnetic isolation. In this step, $400 \mu \mathrm{L}$ of the leukocyte fraction $\left(\sim 5 \times 10^{4}\right.$ leukocytes per $\mu \mathrm{L}$ ) was incubated with the immunomagnetic assays (antibody and magnetic particle suspension) for 10 min, coating the undesired leukocytes with magnetic particles. After incubation, the sample volume was brought to $2.5 \mathrm{~mL}$ by adding Robosep buffer, and the tube containing the cell suspension was placed in the EasySep magnet for $5 \mathrm{~min}$. In this step, the undesired leukocytes were bound to the tube sidewall by the magnetic force, whereas the remaining leukocytes of the desired subtype were poured into a new tube. Finally, the collected leukocytes were washed and resuspended in $20 \mu \mathrm{L}$ phosphate buffered saline (PBS) as the samples of the purified leukocyte subtypes. The purity of these samples were verified using the Wright's staining $(>90 \%)$, and the leukocyte concentrations were measured using a hemacytometer, Bright-Line (Hausser Scientific, USA).

\section{Assay recipe and staining procedure}

The stock solution of the fluorescent dye assay was prepared according to the following procedures. PI stock was prepared by diluting PI solution (1.5 mM, Invitrogen, USA) with PBS to a final concentration of $150 \mu \mathrm{M}$. FITC stock was prepared by first dissolving the dye powder (Invitrogen, USA) in dimethyl sulfoxide (Sigma Aldrich, USA), and then diluted with PBS to a final concentration of $100 \mu \mathrm{M}$. BO21 stock was prepared by dissolving the dye powder (Sigma Aldrich, USA) in PBS to a final concentration of $250 \mu \mathrm{M}$. The dye assay stock (PI, FITC and $\mathrm{BO} 21$ ) was a mixture of these three solutions with a volume ratio of $6: 1: 5$. A modified assay stock (PI and FITC), which replaced the $\mathrm{BO} 21$ solution with equal amount of PBS buffer, was also prepared for comparison studies.
The staining of whole blood samples was carried out according to the following procedure for microflow cytometer measurement. First, $5 \mu \mathrm{L}$ whole blood was incubated with $5 \mu \mathrm{L}$ Cal-Lyse fixation solution (Invitrogen, USA) for 7 min to make the cell membrane permeable. Second, the sample was mixed with $45 \mu \mathrm{L}$ of distilled water for $1 \mathrm{~min}$, lysing erythrocyte cells, and then quenched by adding $6 \mu \mathrm{L} 10 \times$ PBS buffer (Invitrogen, USA). Finally, the sample was incubated with 12 $\mu \mathrm{L}$ of the dye assay (PI, FITC and BO21) for $7 \mathrm{~min}$. For each experiment, $50 \mu \mathrm{L}$ of the stained sample was taken for measurement. The staining recipe contributed to approximately 14 times dilution of the whole blood.

The staining of purified leukocyte subtypes was also carried out for microscope observations. First, the cell suspension of leukocyte subtypes was diluted with PBS to approximately 1000 cell per $\mu \mathrm{L}$. Second, $5 \mu \mathrm{L}$ of the suspension was stained following the same procedure described above, except that in the second step the $45 \mu \mathrm{L}$ of distilled water was replaced by the same amount of PBS. Finally, $8 \mu \mathrm{L}$ of the stained sample was dispensed on a microscope glass slide and spread by a cover slide into a smear for microscope observation.

\section{Results and discussion}

First, leukocyte subtypes were stained with the fluorescent dye assay (PI, FITC and BO21), and the smear samples were imaged using a microscope, LSM510 (Zeiss, Germany), to compare the staining patterns. Fig. 3-a shows the fluorescence images of the leukocyte subtypes including lymphocyte, monocyte, neutrophil and eosinophil. The images were taken by a $100 \times$ objective lens (water immersion) and the fluorescence was measured at two wavelength bands, green $(515-545 \mathrm{~nm})$ and red $(>600 \mathrm{~nm})$. The nuclei of leukocytes showed high intensity of red fluorescence, which was mainly induced by the PI staining. The cytoplasm of the leukocytes
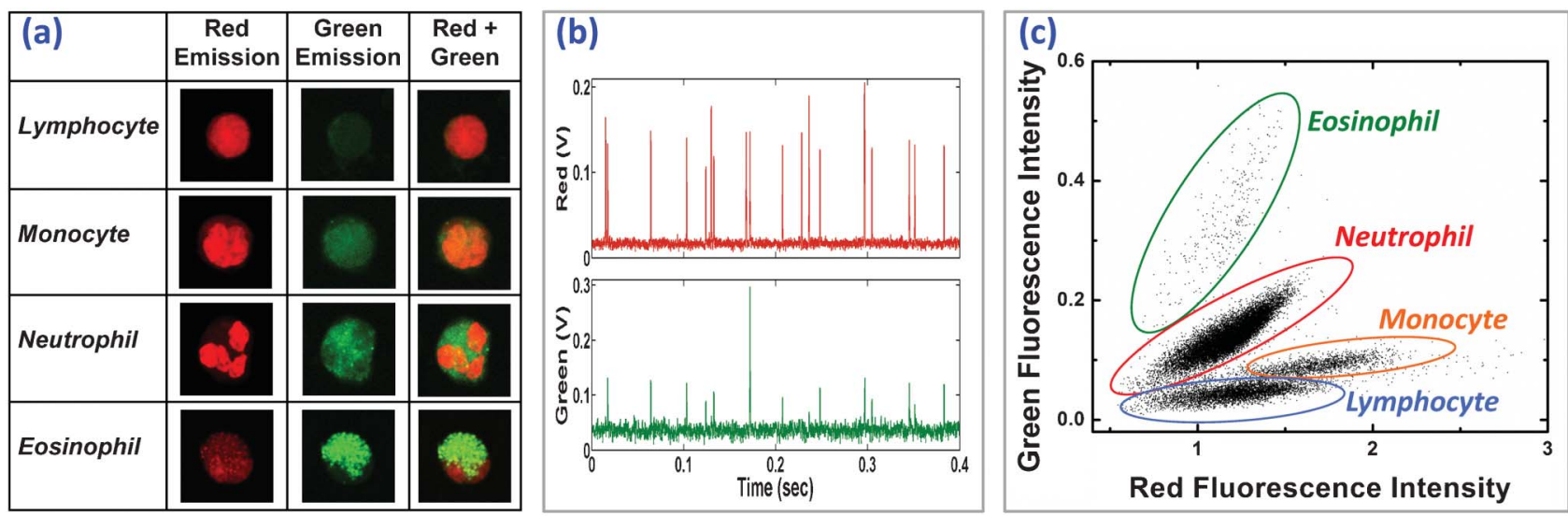

Fig. 3 (a) Fluorescence microscope images of cells of different leukocyte subtypes. The cells were stained with the dye assay (PI, FITC and BO21). Four subtypes

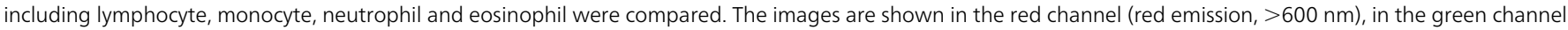

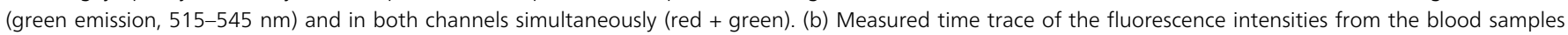
tested on the microflow cytometer. The intensities of the red fluorescence (Red, >600 nm) and the green fluorescence (Green, 520-550 nm) were measured

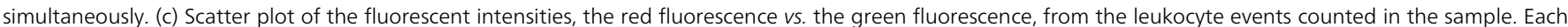

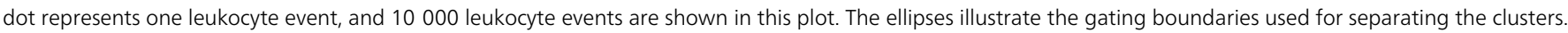


showed high intensity of green fluorescence, which was induced by the FITC staining and the $\mathrm{BO} 21$ staining.

The integrated fluorescence intensities of the cell images were quantified using the software, Zeiss AIM software, ${ }^{34}$ as shown in Fig. S4 ESI. $\dagger$ For the red fluorescence, monocytes had relatively higher intensity than the other three subtypes. For the green fluorescence, eosinophils had highest intensity; neutrophils had median intensity, whereas lymphocytes and monocytes had relatively low intensities. The difference in the red fluorescence was attributed to the selective affinity of PI towards leukocyte nuclei. The nuclei of monocytes have higher PI staining affinity than the nuclei of lymphocytes, neutrophils and eosinophils. ${ }^{35}$ The difference in the green fluorescence was attributed to the different amount of proteins for FITC staining. The granules in leukocyte cytoplasm were rich in proteins. Eosinophils contain the highest amount of granules, which are clearly visible as the sphere-shaped features in the green fluorescence image (Fig. 3-a eosinophil), hence have highest intensity of green fluorescence. Neutrophils have median amount of granules, whereas the lymphocytes and monocytes rarely have any granules. These observations were consistent with a previous report of FITC affinities towards the leukocyte subtypes. ${ }^{36}$ The fluorescence of the leukocytes induced by the dye assay was later measured on the microflow cytometer for both counting the leukocytes and identifying the leukocyte subtypes.

Secondly, PeakFlow Carmine Reference Beads (Invitrogen, USA) was used to calibrate the fluorescence measurement in the sheathless microfluidic channel. The fluidic suspension of the fluorescent beads ( $6 \mu \mathrm{m}$ diameter, emission peak $620 \mathrm{~nm}$ ) was diluted with the PBS buffer to a concentration approximately 200 beads per $\mu \mathrm{L}$. The beads sample was then loaded into the cytometer and measured at the red channel $(>600$ $\mathrm{nm})$. In the measured time trace of the fluorescence signals from the sample, as shown in Fig. S5-a ESI, $†$ each high peak represents one event of the bead being detected in the sheathless channel.

The intensities measured from 4000 bead events (average signal-to-noise ratio $46 \mathrm{~dB}$ ) were fitted by a Gaussian distribution, as shown in Fig. S5-b ESI, $\dagger$ and showed a standard deviation of $8 \%$. The measured variation $(8 \%)$ was higher than the intrinsic variation of the beads $(2 \%)$, which was calibrated on a conventional flow cytometer, FACSCalibur (BD Biosciences, USA). This difference was mainly contributed to the non-uniform intensity of the laser beam (e.g. Gaussian profile) used in the prototype system. To further reduce the variation, beam-shaping optics, such as a Powell lens, could be used to transform the laser from Gaussian profile to Top-Hat profile, which has a much flattened intensity distribution near the beam center. ${ }^{37}$ Nevertheless, this level of variation was tolerable for the leukocyte differential as shown in the following experiments.

Thirdly, whole blood samples were stained with the dye assay (PI, FITC and BO21) and measured on the microflow cytometer. Fig. 3-b shows the measured signals of both the red fluorescence $(>600 \mathrm{~nm})$ and green fluorescence $(520-550 \mathrm{~nm})$.
The width of the fluorescence peaks was approximately $0.5 \mathrm{~ms}$. The average signal-to-noise ratio was $24 \mathrm{~dB}$ for the red fluorescence signal and $14 \mathrm{~dB}$ for the green fluorescence signal. Each high peak of the red fluorescence represents one event of a leukocyte being detected. The heights of the red peak and the corresponding green peak were recorded as the fluorescent intensities of the counted leukocytes.

Fig. 3-c shows a scatter plot of the fluorescence intensities (red versus green) measured from 10000 leukocyte events. In the scatter plot, each dot represents one leukocyte event, and the recorded dots fall into four distinct clusters according to the measured fluorescence intensities. The top cluster was associated with eosinophils, as they had the highest intensities of the green fluorescence. The middle cluster was associated with neutrophils according to their median intensities of the green fluorescence. The bottom two clusters with lowest green intensities were associated with lymphocytes (bottom left) and monocytes (bottom right), whereas the cluster with higher red intensity was associated with monocytes.

The numbers of leukocytes in each cluster were then enumerated to calculate the total leukocyte count and the differential count of each leukocyte subtype. A preliminary gating strategy was used in this study, as shown in Fig. 3-c, where the boundaries between clusters were approximated by ellipses. The total leukocyte count was calculated by dividing the total number of leukocytes by the volume of the blood sample. The differential count was calculated as the percentages of each cluster among the total leukocytes. For the sample of Fig. 3-c, Table 1 summarizes the results from both the test performed on the microflow cytometer and the test performed independently on a state-of-art hematology analyzer (Coulter LH750) in a clinical laboratory (USC Clinical Reference Laboratory, USA). The consistency between the results suggested that counted clusters were indeed mainly associated with the corresponding subtypes.

Correlation study of results from the microflow cytometer and results from the clinical laboratory test was also carried out. Whole blood samples from nine anonymous donors $(n=$ 9) were tested. Two vials of blood were collected from each donor, whereas one vial was tested on the microflow cytometer and the other was tested independently on a hematology analyzer (Coulter LH750). Three repeated measurements were performed on the microflow cytometer for each sample and the average values were used as the test results.

Fig. 4-a shows the correlation of total leukocyte count between the microflow cytometer and the clinical laboratory test. For the measured range of 4000-8000 leukocytes per $\mu \mathrm{L}$, the test results showed a close to linear correlation (linear regression slope 0.97 , correlation coefficient $R^{2}=0.97$ ). In addition, the maximum inaccuracy (defined as the difference between the cytometer data and the clinical laboratory data) for the tested samples $(n=9)$ was less than $10 \%$, which was within the allowable error for clinical significance (16.5\%). ${ }^{1}$

Fig. 4-b shows the correlations of the differential count results for each of the four subtypes including lymphocyte, monocyte, neutrophil and eosinophil. The measured percen- 
Table 1 Comparison of the leukocyte count results of the same sample from the test on the microflow cytometer and the clinical laboratory test on a hematology analyzer Coulter LH750

\begin{tabular}{llllll} 
& & \multicolumn{2}{l}{ Four-part differential $(\%)$} & \\
\cline { 3 - 5 } & Total count $\left(\right.$ cells $\left.\mu \mathrm{L}^{-1}\right)$ & Lymphocyte & Monocyte & Neutrophil & Eosinophil \\
\hline Microflow cytometer & 6400 & 22.5 & 7.8 & 68.3 & 1.4 \\
Coulter LH750 & 6900 & 21.6 & 8.0 & 69.2 & 1.2
\end{tabular}

tages of the two major subtypes, lymphocyte (15-35\% of total leukocytes) and neutrophil (50-75\% of total leukocytes), showed close to linear correlations (linear regression slopes $S=1.00-1.03$ ) with a high correlation coefficient $R^{2}=0.97-$ 0.98. The percentages of the rare subtype, eosinophil (1-4\% of total leukocytes), also showed a close to linear correlation $(S=$ $1.04)$, although the correlation coefficient is slightly lower $\left(R^{2}=\right.$ 0.93). The percentages of the monocyte $(5-12 \%$ of total leukocytes) showed the lowest but still satisfactory correlation $\left(R^{2}=0.90\right)$, and the linear regression slope $S=0.91$ indicated that the counted monocyte percentages on the microflow cytometer were slightly lower than the percentages counted on the hematology analyzer. Overall, the test results from the microflow cytometer matched the clinical laboratory test with a maximum inaccuracy less than $10 \%$, which was within the tolerable error for clinical significance $15 \%$ to $26 \%$ for the four subtypes respectively ${ }^{1}$ ).

The observed close correlations for the leukocyte differential count confirmed that the clusters in the scatter plot indeed mainly consisted of the corresponding leukocyte subtypes. Nevertheless, the associations between the clusters and the subtypes were further validated with spiking experiments. For each of the four subtypes, an experiment sample (3 $\mu \mathrm{L}$ whole blood spiked with $2 \mu \mathrm{L}$ purified cell suspension of the target subtype) and a control sample ( $3 \mu \mathrm{L}$ whole blood mixed with $2 \mu \mathrm{L}$ PBS buffer) were both measured on the microflow cytometer. The measured scatter plots of the samples were then compared in Fig. 5. The target clusters in the spiked samples (Fig. 5-d to Fig. 5-e) were denser and more significant than their counterparts in the control sample (Fig. 5-a). These changes were particularly obvious for clusters that were sparse in the control sample, such as the eosinophil cluster and the monocyte cluster.

Table 2 summarizes the measured count of the target clusters before spiking, the expected count from spiking (assuming 100\% of the spiked cell measured in the target cluster), and the measured count after spiking. For each of the target clusters, the measured count indeed increased significantly after spiking. For instance, the differential count of the eosinophil subtype consisted of only $2.1 \%$ of the total leukocytes before spiking, but reached as high as $14.0 \%$ (7 times increase) after spiking. This change confirmed that the spiked cells indeed led to the increased count of the target cluster. In addition, the measured counts after spiking matched closely with the counts expected from the number of cell spiked, e.g. for clusters of eosinophil (measured 14.0\%,
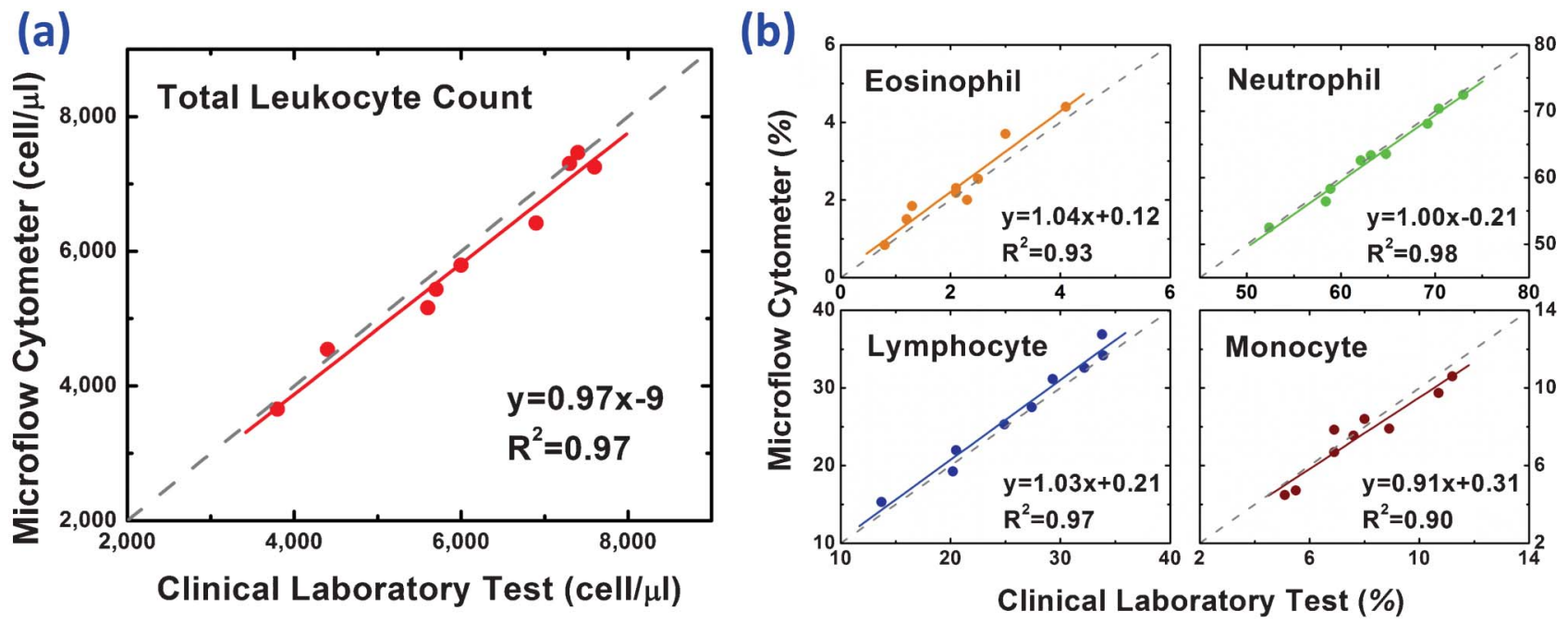

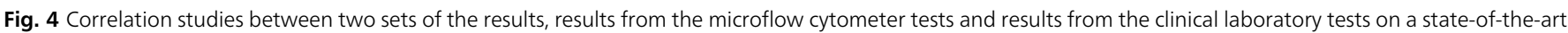

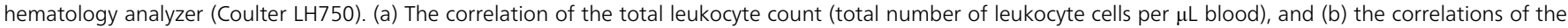

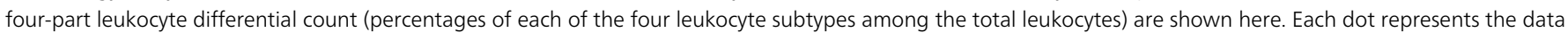

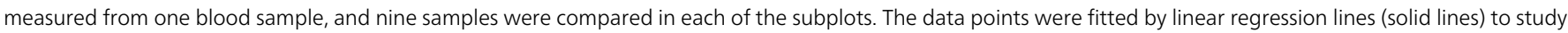

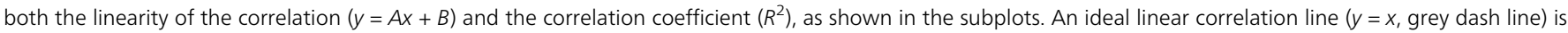
also shown in each of the subplots for comparison. 

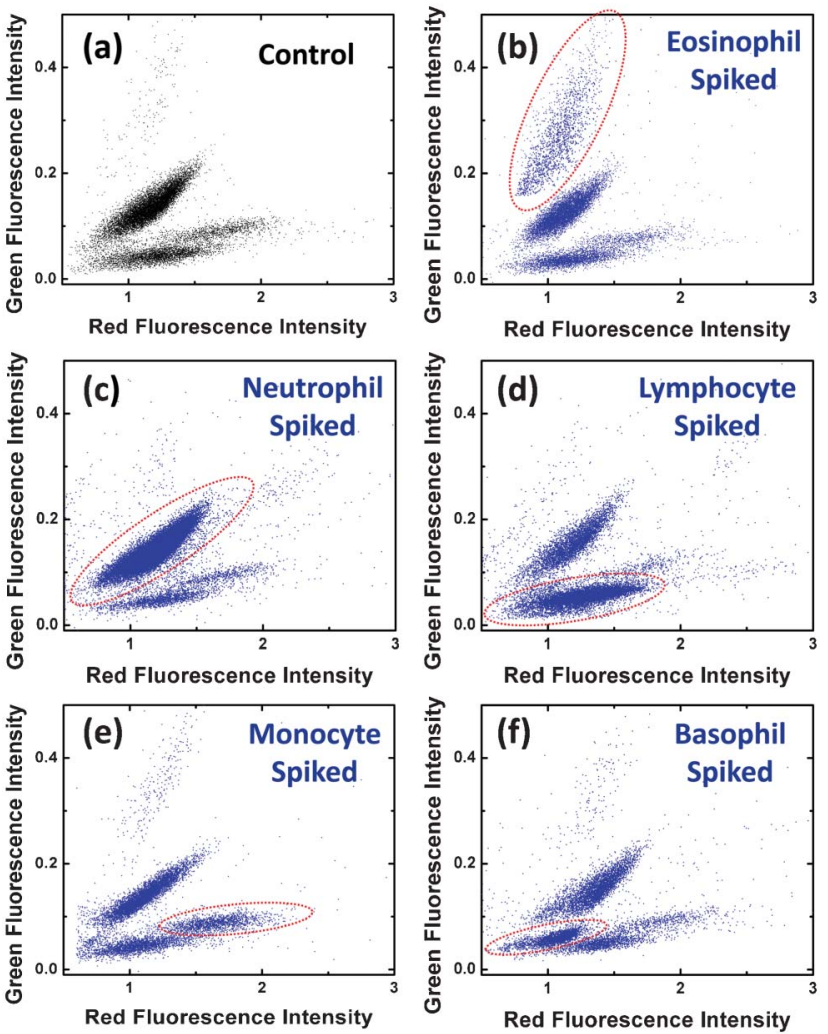

Fig. 5 Results of the scatter plots from the spiking experiments. (a) The control sample, and samples spiked with purified leukocyte subtypes, (b) eosinophils, (c) neutrophils, (d) lymphocytes, (e) monocytes and (f) basophils. The target clusters in the spiking experiments $(b-f)$ are illustrated by the red dashed ellipses.

expected 14.4\%), neutrophil (measured 89.2\%, expected $90.3 \%$ ) and lymphocyte (measured $63.9 \%$, expected $66.5 \%$ ). These results confirmed that the spiked cells mostly fell into these target clusters. The measured count of the monocyte cluster $(16.7 \%)$ was relatively lower than the expected count (21.2\%), suggesting some of the spiked monocytes did not fall into the designated monocyte cluster.

The count of monocyte is less satisfactory than the counts of the other three subtypes (lymphocyte, neutrophil and eosinophil) even on the state-of-the-art hematology analyzers. ${ }^{1}$ The count of monocyte has been investigated in a preliminary study we reported. In that study, a different dye assay (PI and FITC) was used, in which the monocyte cluster was partially overlapping with the lymphocyte cluster. $^{24}$ In the newly proposed assay of this study (PI, FITC and BO21), the dye BO21 was hence added to improve the monocyte count. The improvement of the monocyte count was demonstrated in the following experiments. Two samples of $5 \mu \mathrm{l}$ whole blood from the same vial were stained by the assay without BO21 (PI and FITC) and the assay with $\mathrm{BO} 21$ (PI, FITC and $\mathrm{BO} 21$ ) respectively, and measured on the microflow cytometer.

The scatter plots measured from these two assays are compared in Fig. S6, ESI. $\dagger$ In the assay without BO21, as shown in Fig. S6-a, ESI, $\dagger$ the green intensity of the monocyte cluster was similar to the green intensity of the lymphocyte cluster, and these two clusters were partially overlapping with each other (Fig. S6-b, ESI $\dagger$ ). This overlap led to the relatively poorer accuracy of the monocyte count (linear regression slope $S=$ 0.50 , correlation coefficient $R^{2}=0.76$ ) as shown in a correlation study with the central laboratory test. ${ }^{24}$ In the newly proposed assay with BO21, as shown in Fig. S6-c, ESI, $\dagger$ the monocyte cluster had a significantly higher green fluorescence intensity than the lymphocyte cluster, leading to a clear separation of these two clusters (Fig. S6-d, ESI†). The improvement of the count accuracy was evident as shown in the correlation study above $\left(S=0.91, R^{2}=0.90\right)$.

Above all, the four-part differential count of this study classified leukocytes into four subtypes including lymphocytes, monocytes, neutrophils and eosinophils. In addition to these four subtypes, a fifth subtype, basophil is also reported in clinical laboratory tests for the complete five-part differential count. However, the basophils are normally rare $(<1 \%$ of total leukocytes), and the accuracy of the basophil count is questionable even on state-of-the-art hematology analyzers. ${ }^{1}$ Nevertheless, the count of basophil was investigated in this assay to demonstrate the feasibility of achieving the complete five-part differential.

In human whole blood, the basophil count is normally low (less than $1 \%$ of total leukocytes). Therefore, the basophil count was studied using the spiking experiment. Two samples were measured, including a sample of whole blood spiked with the purified basophils and a control sample without the spiking. The measured scatter plot of the spiked sample, as

Table 2 The differential count results of the target clusters in the spiking experiments. Tests 1-5 each correspond to the samples spiked with the eosinophils, neutrophils, lymphocytes, monocytes and basophils. The control sample was whole blood without spiking. The "Reference" data was measured in the clinical laboratory test. The spiked samples were whole blood spiked with known number of the purified subtypes. The "Expected" data was calculated based on the

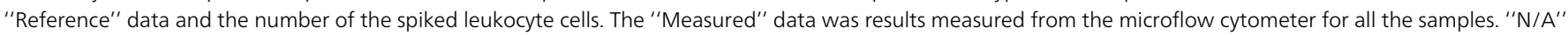
means the count result is not available

\begin{tabular}{|c|c|c|c|c|c|}
\hline \multirow[b]{2}{*}{ Test } & \multirow[b]{2}{*}{ Target cluster } & \multicolumn{2}{|l|}{ Control sample } & \multicolumn{2}{|c|}{ Spiked samples } \\
\hline & & Reference (\%) & Measured (\%) & Expected (\%) & Measured (\%) \\
\hline 1 & Eosinophil & 2.1 & 2.1 & 14.4 & 14.0 \\
\hline 2 & Neutrophil & 69.2 & 68.1 & 90.3 & 89.2 \\
\hline 3 & Lymphocyte & 20.5 & 22.0 & 66.5 & 63.9 \\
\hline 4 & Monocyte & 6.9 & 7.3 & 21.2 & 16.7 \\
\hline 5 & Basophil & 0.4 & $\mathrm{~N} / \mathrm{A}$ & 32.0 & 29.3 \\
\hline
\end{tabular}


shown in Fig. 5-f, had a fifth cluster in addition to the four clusters in the control sample, as shown in Fig. 5-a. The count of this cluster $(29.3 \%)$, as summarized in Table 2, matched closely with the count expected from the spiked amount of purified basophils (32\%), suggesting this cluster mainly consisted of basophils. The scatter plot of the control sample (Fig. 5-a) did not form a distinct basophil cluster mainly due to the low basophil count in the whole blood sample $(0.4 \%$ as measured in the central laboratory test). These results demonstrated that the reported method of the dye assay and the microflow cytometer could be used in clinical tests to flag abnormal samples with a high basophil count.

\section{Conclusions}

This paper described a leukocyte count with a four-part differential (lymphocyte, monocyte, neutrophil and eosinophil) using a fluorescent dye assay and a sheathless microflow cytometer. The dye assay (PI, FITC and BO21) selectively stained leukocytes in blood samples, and the sheathless microflow cytometer measured the fluorescence from the sample to count leukocytes. Leukocytes were counted by their high intensity of the red fluorescence $(>600 \mathrm{~nm})$ and the leukocyte subtypes were identified by the measured intensities of the red fluorescence and the green fluorescence (520-550 $\mathrm{nm})$.

The count results measured on the cytometer showed close correlation with results measured from clinical laboratory tests (sample size $n=9$ ). The total leukocyte count showed a linear correlation with a linear regression slope $S=0.97$ and a correlation coefficient $R^{2}=0.97$, and a count inaccuracy less than $10 \%$. The differential count also showed linear correlation for each of the four subtypes (lymphocyte $S=1.03, R^{2}=$ 0.97; monocyte $S=0.91, R^{2}=0.90$; neutrophil $S=1.00, R^{2}=$ 0.98; eosinophil $S=1.04, R^{2}=0.93$ ).

The reported method consumed only a small amount of blood sample $(5 \mu \mathrm{L})$ and minimal volume of reagent $(68 \mu \mathrm{L}$ in total). In addition, no sheath buffer was needed for the microflow cytometer. The complete system was packaged in a portable size $\left(12^{\prime \prime} \times 9^{\prime \prime} \times 5^{\prime \prime}\right.$ for the prototype of this study), and suitable for point-of-care applications.

\section{Acknowledgements}

This work was supported by NSBRI under NASA contract NCC9-58. The authors would like to thank Shijia Chen for the measurement on the scanning microscope. The authors also would like to thank all members of Caltech Micromachining Group for their helpful discussions.

\section{References}

1 M. Buttarelo and M. Plebani, Am. J. Clin. Pathol., 2008, 130, 104-116.
2 D. Heikali and D. Di Carlo, J. Assoc. Lab. Autom., 2010, 15, 319-328.

3 S. Epelman and D. R. Hamilton, Aviation, Space and Environmental Medicine, 2006, 77, 130-139.

4 E. Altendorf, D. Zebert, M. Holl and P. Yager, Proceeding of 1997 International Conference on Solid-State Sensors and Actuators, 1997, 1, 531-534.

5 S. Seo, S. O. Isikman, I. Sencan, O. Mudanyali, T. Su, W. Bishara, A. Erlinger and A. Ozcan, Anal. Chem., 2010, 82, 4621-4627.

6 D. Holmes, D. Pettigrew, C. H. Reccius, J. D. Gwyer, C. van Berkel, J. Holloway, D. E. Davies and H. Morgan, Lab Chip, 2009, 9, 2881-2889.

7 T. D. Chung and H. C. Kim, Electrophoresis, 2007, 28, 4511-4520.

8 S. D. H. Chan, G. Luedke, M. Valer, C. Buhlmann and T. Preckel, Cytometry, Part A, 2003, 55, 119-125.

9 J. H. Wang, C. H. Wang, C. C. Lin, H. Y. Lei and G. B. Lee, Microfluid. Nanofluid., 2011, 10, 531-541.

10 H. Yun, H. Bang, J. Min, C. Chung, J. K. Chang and D. C. Han, Lab Chip, 2010, 10, 3243-3254.

11 J. K. Herr, J. P. Alarie, J. Soohoo, G. M. Walker, N. Sharpless and J. M. Ramsey, Proceeding of the 12th International Conference on Miniaturized Systems for Chemistry and Life Sciences, 2008, 1102-1104.

12 A. Kummrow, J. Theisen, M. Frankowski, A. Tuchscheerer, H. Yildirim, K. Brattke, M. Schmidt and J. Neukammer, Lab Chip, 2009, 9, 972-981.

13 M. Frankowski, N. Bock, A. Kummrow, S. Schädel-Ebner, M. Schmidt, A. Tuchscheerer and J. Neukammer, Cytometry, Part A, 2011, 79, 613-624.

14 W. Hubl, G. Wolfbauer, S. Andert, G. Thum, J. Streicher, C. Hubner, A. Lapin and P.M. Bayer, Cytometry, 1997, 30, 72-84.

15 D. Huh, W. Gu, Y. Kamotani, J. B. Grotberg and S. Takayama, Physiol. Meas., 2005, 26, R73-R98.

16 D. A. Ateya, J. S. Erickson, P. B. Howell, Jr., L. R. Hilliard, J. P. Golden and F. S. Ligler, Anal. Bioanal. Chem., 2008, 391, 1485-1498.

17 J. P. Golden, J. S. Kim, J. S. Erickson, L. R. Hilliard, P. B. Howell, G. P. Anderson, M. Nasir and F. S. Ligler, Lab Chip, 2009, 9, 1942-1950.

18 B. K. Mckenna, A. A. Selim, F. R. Bringhurst and D. J. Ehrlich, Lab Chip, 2009, 9, 305-310.

19 X. Mao, S. S. Lin, C. Dong and T. J. Huang, Lab Chip, 2009, 9, 1583-1589.

20 S. C. Hur, H. T. K. Tse and D. Di Carlo, Lab Chip, 2010, 10, 274-280.

21 H. M. Shapiro, Practical Flow Cytometry, Wiley-Liss, 4th edn, 2003.

22 S. Zheng, J. C.-H. Lin, H. L. Kasdan and Y.-C. Tai, Sens. Actuators, B, 2008, 132, 558-567.

23 W. Shi, S. Zheng, H. L. Kasdan, A. Fridge and Y.-C. Tai, Proceeding of 2009 International Conference on Solid-State Sensors and Actuators, 2009, 616-619.

24 W. Shi, H. L. Kasdan, A. Fridge and Y.-C. Tai, Proceeding of 23th International Conference on Micro Electro Mechanical Systems, 2010, 1019-1022.

25 W. Shi, L. W. Guo, H. Kasdan, A. Fridge and Y.-C. Tai, Proceeding of the 16th International Conference on Solid-State Sensors and Actuators, 2011, 2956-2959. 
26 H. M. Shapiro, J. Histochem. Cytochem., 1977, 25, 976-989. 27 L. R. Adams and L. A. Kamentsky, Acta Cytologica, 1971, 15, 289-291.

28 H. M. Shapiro, E. R. Schildkraut, R. Curbelo, C. W. Laird, R. B. Turner and T. Hirschfeld, J. Histochem. Cytochem., 1976, 21, 396-411.

29 B. J. Bain, Blood Cells: A Practical Guide, Wiley-Blackwell, 4th edn, 2006.

30 G. Mazzini, P. Giordano, A. Riccardi and C. M. Montecucco, Cytometry, 1983, 3, 443-448.

31 J. S. Miller and J. M. Quarles, Cytometry, 1990, 11, 667-675. 32 W. B. Mercer, Rev. Sci. Instrum., 1966, 37, 1515-1520.
33 B. F. Gibbs, K. Papenfuss and F. H. Falcone, Clin. Exp. Allergy, 2008, 38, 480-485.

34 N. Furuta, N. Fujita, T. Noda, T. Yoshimori and A. Amano, Mol. Biol. Cell, 2010, 21, 1001-1010.

35 Z. Darzynkiewicz and X. Huang, Current Protocols in Immunology, 2004, 60, 5.7.1-5.7.18.

36 E. Bedner, H. D. Halicka, W. Cheng, T. Salomon, A. Deptala, W. Gorczyca, M. R. Melamed and Z. Darzynkiewicz, Cytometry, 1999, 36, 77-82.

37 O. Homburg and T. Mitra, Laser Resonators, Microresonators, and Beam Control XIV, Proceeding of the SPIE, 2012, 8236, 82360A-9. 